\title{
Independence of Containing Patterns Property and its Application in Tree Pattern Query Rewriting Using Views
}

\author{
Junhu Wang · Jeffrey Xu Yu • Chengfei Liu
}

Received: / Accepted: date

\begin{abstract}
We show that several classes of tree patterns observe the independence of containing patterns property, that is, if a pattern is contained in the union of several patterns, then it is contained in one of them. We apply this property to two related problems on tree pattern rewriting using views. First, given view $V$ and query $Q$, is it possible for $Q$ to have an equivalent rewriting using $V$ which is the union of two or more tree patterns, but not an equivalent rewriting which is a single pattern? This problem is of both theoretical and practical importance because, if the answer is no, then, to find an equivalent rewriting of a tree pattern using a view, we should use more efficient methods, such as the polynomial time algorithm of [20], rather than try to find the union of all contained rewritings (which takes exponential time in the worst case) and test its equivalence to $Q$. Second, given a set $S$ of views, we want to know under what conditions a subset $S^{\prime}$ of $S$ is redundant in the sense that for any query $Q$, the contained rewritings of $Q$ using the views in $S^{\prime}$ are contained in those using the views in $S-S^{\prime}$. Solving this problem can help us to, for example, choose the minimum number of views to be cached, or better design the virtual schema in a mediated data integration system, or avoid repeated calculation in query optimization. For the first problem, we identify several classes of tree patterns for which the equivalent rewriting can be expressed as a single tree pattern. For the second problem, we present necessary and sufficient conditions for $S^{\prime}$ to be redundant with respect to some classes of tree patterns. For both problems we consider extension to cases where there are rewritings using the intersection of multiple views and/or where a schema graph is present.
\end{abstract}

J. Wang

Griffith University, Gold Coast, Australia

E-mail: J.Wang@griffith.edu.au

J. X. Yu

Chinese University of Hong Kong, Hong Kong, China

E-mail: yu@se.cuhk.edu.hk

C. Liu

Swinburne University of Technology, Melbourne, Australia

E-mail: cliu@ict.swin.edu.au 


\section{Introduction}

Query rewriting using views has many applications including data integration, query optimization, and query caching [5]. A view is an existing query whose answer may or may not have been materialized. Given a new query, the problem is to find another query using only the views that will produce correct answers to the original query. Usually two types of rewritings are sought: equivalent rewritings and contained rewritings. An equivalent rewriting produces all answers to the original query, while a contained rewriting may produce only part of the answers. Both types of rewritings have been extensively studied in the relational database context, see [5] for an early survey, and $[17,12]$ for more recent developments.

More recently rewriting XML queries using XML views has attracted attention because of the rising importance of XML data $[20,9,6,13]$. Since XPATH lies in the center of all XML languages, the problem of rewriting XPATH queries using XPATH views is particularly important. Some major classes of XPATH expressions can be represented as tree patterns $[1,10]$. Among previous work on rewriting XPATH queries using views, $\mathrm{Xu}$ et al [20] studied equivalent rewritings for several different classes of tree patterns, and it gave a polynomial time algorithm for finding equivalent rewritings when the tree patterns do not have *. Mandhani and Suciu [9] presented results on equivalent rewritings of tree patterns when the tree patterns are assumed to be minimized. Lakshmanan et al [6] studied maximal contained rewritings of tree patterns where both the view and the query involve /,// and [] only (these ХРATH expressions correspond to tree patterns in $P^{\{/, / /,[]\}}[10]$ ), both in the absence and presence of non-recursive schema graphs - a restricted form of DTDs. When there are no DTDs, the worst case complexity of finding the maximal contained rewriting is shown to be exponential in the size of the query.

In this paper, we study two related problems on XPATH rewritings using views. The first problem is about the form of equivalent rewritings: given view $V$ and query $Q$, is it possible for $Q$ to have an equivalent rewriting using $V$ which is the union of two or more tree patterns, but not an equivalent rewriting which is a single tree pattern? This problem is of both theoretical and practical importance because, if the answer is no, then, to find an equivalent rewriting using the view, we can use more efficient methods such as the polynomial time algorithm of [20], rather than try to find the union of all contained rewritings [6] and test its equivalence to $Q$. The second problem is what we call the redundant views problem. Given a set $S$ of views, we want to know under what conditions a subset $S^{\prime}$ of $S$ is redundant in the sense that for any query $Q$, the contained rewritings of $Q$ using the views in $S^{\prime}$ are contained in those using the views in $S-S^{\prime}$. Thus the contribution of redundant views to the contained rewritings can be ignored. Solving this problem can help us to, for example, choose the minimum number of views to be cached, or better design the virtual schema in a mediated data integration system, or avoid useless computation in query optimization. We first study the above problems for the class of tree patterns involving /,// and [], and then extend our results to other classes of tree patterns. We also consider the case where the intersection of views is used in the rewriting, as well as the case a schema graph is present.

Our main contributions are: 


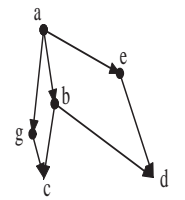

(a) $G_{1}$

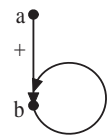

(b) $G_{2}$

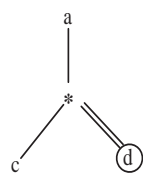

(c) $P_{1}$

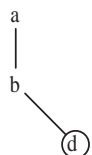

(d) $P_{2}$

Fig. 1. Schema graphs $G_{1}, G_{2}$ and tree patterns $P_{1}, P_{2}$

- We show that, when there is no DTD, several classes of tree patterns observe the independence of containing patterns property, that is, if a tree pattern is contained in the union of multiple tree patterns, then it must be contained in one of them.

- Using the above property, we show that for queries and views in $P^{\{/, / /,[]\}}$, if there is no equivalent rewriting in the form of a single tree pattern, then there is no equivalent rewriting in the form of a union of tree patterns. We extend this result to queries in $P^{\{/,[], *\}}$, queries and views in $\widehat{P}^{\{/, / /,[], *\}}$, and rewritings using multiple views. We also consider the presence of DTDS.

- When multiple views exist, we provide a necessary and sufficient condition for identifying redundant views. In the case where the intersection of views is also used in the rewriting, we provide a necessary condition and a separate sufficient condition for redundant views.

The rest of the paper is organized as follows. Section 2 provides the terminology and notations. Section 3 shows the independence of containing patterns property of tree patterns. Based on this property, Section 4 presents our result on the form of equivalent rewritings. Section 5 then discusses conditions under which some views are redundant. Section 6 discusses related work. Finally Section 7 concludes the paper.

\section{Preliminaries}

2.1 DTDs, XML trees, and tree patterns

Let $\Sigma$ be an infinite set of tags. We adopt the similar notations used in [6], and model a DTD as a connected directed graph $G$ (called a schema graph) satisfying the following conditions:

(1) each node is labeled with a distinct label in $\Sigma$;

(2) each edge is labeled with one of $1, ?,+$, and $*$, which indicate "exactly one", "one or zero", "one or many", and "zero or many", respectively. Here, the default edge label is $*$;

(3) there is a unique node, called the root, which may have an incoming degree of zero. All other nodes have incoming degrees greater than 0 .

Because a node in a schema graph $G$ has a unique label, we also refer to a node by its label. If the graph is acyclic, then the DTD is said to be non-recursive. We will use DTD and schema graph interchangeably ${ }^{1}$. Two example schema graphs are shown in Fig. 1 (a) and Fig. 1 (b), the first one is non-recursive, and the second one is recursive.

\footnotetext{
1 A schema graph cannot model constructs such as $a:=\left(b, b^{?}\right)$ and $a:=(b \mid c)$ in a DTD.
} 
An XML tree is a node-labeled, unordered tree. Let $v$ be a node in an XML tree $t$, the label of $v$ is denoted by label $(v)$. Let $N(t)$ (resp. $N(G)$ ) denote the set of all nodes in XML tree $t$ (resp. schema graph $G$ ), and $r t(t)$ (resp. $r t(G)$ ) denote the root of $t$ (resp. $G)$. A tree $t$ is said to conform to schema graph $G$ if

(1) for every node $v \in N(t), \operatorname{label}(v) \in \Sigma$,

(2) $\operatorname{label}(r t(t))=\operatorname{label}(r t(G))$,

(3) for every edge $(u, v)$ in $t$, there is a corresponding edge $(\operatorname{label}(u), \operatorname{label}(v))$ in $G$, and

(4) for every node $v \in N(t)$, the number of children of $v$ labeled with $x$ is constrained by the label of the edge $(\operatorname{label}(v), x)$ given in $G$.

We denote the set of all XmL trees conforming to $G$ by $T_{G}$.

We consider a class of XPATH expressions given as follows.

$$
P \quad::=\tau|*| P / P|P / / P| P[P] \mid P[/ / P]
$$

Here, $\tau \in \Sigma, *$ is the wildcard representing any tag in $\Sigma$, and, /, //, and [] represent the child-axis, descendant-axis, and branching condition, respectively. The class of XPATH expressions corresponds to a set of tree patterns (TP) known as $P^{\{/, / /,[], *\}}$ in [10]. Formally, a tree pattern (TP) in $P^{\{/, / /,[], *\}}$ is a tree such that each edge is labeled with either / or //; each node is labeled with a tag in $\Sigma$ or the wildcard $*$, and there is a distinguished node corresponding to the output of the XPath expression. When there is no confusion, we will use TP and XPath query interchangeably. A tree patten has a tree representation. Fig. 1 (c) and (d) show two TPs. They correspond to the XPATH expressions $a / *[c] / / d$ and $a / b / d$, respectively. Here, single lines represent edges labeled with /, called /-edges, and double lines represent edges labeled with //, called //-edges. A branch in the tree representation represents a condition ([]) in an XPATH expression, and a circle indicates the distinguished node of $P$. Below, given a TP $P$, we use $\mathrm{DN}_{P}$ to denote the distinguished node. The path from $r t(P)$ to $\mathrm{DN}_{P}$ is called the distinguished path.

The following subsets of $P^{\{/, / /,[], *\}}$ are of special interest to us. $P^{\{/, / /,[]\}}$is the set of TPs that do not have ${ }^{*}$-nodes (i.e., nodes labeled with $\left.{ }^{*}\right), P^{\{/,[], *\}}$ is the set of TPs that do not have //-edges, and $\widehat{P}^{\{/, / /,[], *\}}$ is the set of TPs such that no //-edge is incident on a *-node, and there are no leaf *-nodes. Note that $P^{\{/, / /,[]\}}$is a subset of $\widehat{P}^{\{/, / /,[], *\}}$.

Let $N(P)$ (resp. $r t(P)$ ) denote the set of all nodes in a TP $P$ (resp. the root of $P$ ). A matching of $P$ in an xML tree $t$ is a mapping $\delta$ from $N(P)$ to $N(t)$ which is

(1) root-preserving, i.e., $\delta(r t(P))=r t(t)$,

(2) label-preserving, i.e., $\forall v \in N(P)$, label $(v)=\operatorname{label}(\delta(v))$ or $\operatorname{label}(v)=*$, and

(3) structure-preserving, i.e., for every edge $(x, y)$ in $P$, if it is a /-edge, then $\delta(y)$ is a child of $\delta(x)$; if it is a //-edge, then $\delta(y)$ is a descendant of $\delta(x)$, i.e, there is a path from $\delta(x)$ to $\delta(y)$

Each matching $\delta$ produces a subtree of $t$ rooted at $\delta\left(\mathrm{DN}_{P}\right)$, denoted $s u b_{\delta\left(\mathrm{DN}_{P}\right)}^{t}$, which is also known as an answer to the TP. We use $P(t)$ to denote the set of all answers of $P$ on $t$ :

$$
P(t)=\left\{s u b_{\delta\left(\mathrm{DN}_{P}\right)}^{t} \mid \delta \text { is a matching of } P \text { in } t\right\}
$$

If $P(t)=\emptyset$ for every xmL tree $t$, then $P$ is said to be an empty query, denoted $P(t)=\emptyset$. 
Let $T$ be a set of xmL trees. We use $P(T)$ to denote the union of answer sets of $Q$ on the trees in $T$. That is, $P(T)=\bigcup_{t \in T} P(t)$. In addition, when we discuss TPs in the presence of DTD $G$, we will implicitly assume every TP $P$ is satisfiable under $G$, that is, there is $t \in T_{G}$ such that $P(t) \neq \emptyset$.

2.2 Tree pattern containment and containment mapping

A TP $P$ is said to be contained in another TP $Q$, denoted $P \subseteq Q$, if for every xML tree $t, P(t) \subseteq Q(t)$ (Refer to Eq. (1)). Given a DTD $G$ and two TPs $P$ and $Q, P$ is said to be contained in $Q$ under $G$, denoted $P \subseteq_{G} Q$, if for every xmL tree $t \in T_{G}, P(t) \subseteq Q(t)$. Tree pattern equivalence is defined as two-way containment as usual. That is, $P=Q$ is defined as $P \subseteq Q$ and $Q \subseteq P$, and $P=_{G} Q$ means $P \subseteq_{G} Q$ and $Q \subseteq_{G} P$.

When there are no DTDS, the containment of some classes of tree patterns can be characterized by the existence of a containment mapping. Recall [1]: a containment mapping (CM) from $Q$ to $P$ is a mapping $h$ from $N(Q)$ to $N(P)$ that is label-preserving, root-preserving, structure-preserving (which now means that for every /-edge $(x, y)$ in $Q,(h(x), h(y))$ is a /-edge in $P$, and for every //-edge $(x, y)$, there is a path from $h(x)$ to $h(y))$, and output-preserving, which means $h\left(\mathrm{DN}_{Q}\right)=\mathrm{DN}_{P}$. The following lemma is proved in [11].

Lemma 1 [11] In the following cases, $P \subseteq Q$ iff there is a $\mathrm{CM}$ from $Q$ to $P$.

(1) $Q \in P^{\{/, / /,[]\}}$

(2) $P \in P^{\{/,[], *\}}$,

(3) $Q \in P^{\{/,[], *\}}$, and there are no leaf ${ }^{*}$-nodes in $Q$.

A closer inspection of the proof in [11] shows the following lemma is also true.

Lemma 2 Suppose $Q \in \widehat{P}^{\{/, / /,[], *\}}$. Then for any pattern $P \in P^{\{/, / /,[], *\}}, P \subseteq Q$ iff there is a $\mathrm{CM}$ from $Q$ to $P$.

2.3 Contained rewriting, maximal contained rewriting and equivalent rewriting

A view is a pre-defined TP. Let $V$ be a view and $Q$ be a TP. A contained rewriting (CR) of $Q$ using $V$ is a TP $Q^{\prime}$ such that when evaluated on the subtrees returned by $V$, $Q^{\prime}$ gives correct answers to $Q$. More precisely, (1) for any xML tree $t, Q^{\prime}(V(t)) \subseteq Q(t)$, and (2) there exists some $t$ such that $Q^{\prime}(V(t)) \neq \emptyset$.

Let $Q^{\prime}$ be a CR of $Q$. We use $Q^{\prime} \circ V$ to represent the expansion of $Q^{\prime}$, which is the TP obtained by merging $r t\left(Q^{\prime}\right)$ and $\mathrm{DN}_{V}$ as follows: if $\operatorname{label}\left(\mathrm{DN}_{V}\right) \neq *$, then the merged node is labeled label $\left(\mathrm{DN}_{V}\right)$, otherwise the merged node is labeled label $\left(r t\left(Q^{\prime}\right)\right)$ (Note that if label $\left(\operatorname{DN}_{V}\right) \neq *$, label $\left(r t\left(Q^{\prime}\right)\right) \neq *$ then label $\left.\left(r t\left(Q^{\prime}\right)\right)=\operatorname{label}\left(\operatorname{DN}_{V}\right)\right)$. The distinguished node of $Q^{\prime} \circ V$ is the distinguished node of $Q^{\prime}$. Fig.2 shows a view $V$, a TP $Q$, a CR $Q^{\prime}$ of $Q$ using $V$, and the expansion $Q^{\prime} \circ V$ of $Q^{\prime}$.

It is easy to see that $\left(Q^{\prime} \circ V\right)(t)=Q^{\prime}(V(t))$. Thus condition (1) in the definition of CR is equivalent to $Q^{\prime} \circ V \subseteq Q$, and condition (2) in the definition is equivalent to $Q^{\prime} \circ V \neq \emptyset$. A CR $Q^{\prime}$ is said to be an equivalent rewriting (ER) if $Q^{\prime} \circ V \supseteq Q$ also holds. The maximal contained rewriting $(\mathrm{MCR})$, denoted $\operatorname{MCR}(Q, V)$, is the union of all CRs of $Q$ using $V$ [6]. We use $\operatorname{EMCR}(Q, V)$ to denote the union of expansions of all 

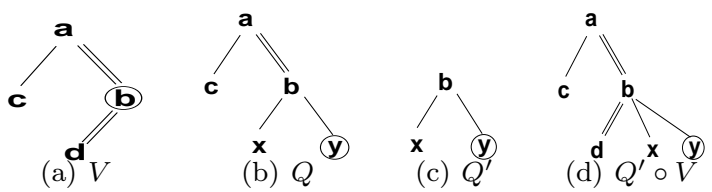

Fig. 2. View (a), Query (b), CR (c), and Expansion (d)

of the CRs in $\operatorname{MCR}(Q, V)$. Using the concepts of useful embedding [6] and revised useful embedding [19], it can be easily proved that, when $Q$ is in $P^{\{/ / / /,[]\}}$or $\widehat{P}^{\{/, / /,[], *\}}$, the MCR of $Q$ using $V$ is the union of a finite number of CRs of $Q$ using $V$. That is, there are CRs $Q_{1} \ldots Q_{m}$ such that $\operatorname{EMCR}(Q, V)=Q_{1} \circ V \cup \cdots \cup Q_{m} \circ V$. For convenience, we define $\operatorname{EMCR}(Q, V)$ to be the empty query if $Q$ has no CRs using $V$.

In the presence of DTDs, a contained rewriting (resp. equivalent rewriting) of $Q$ using view $V$ under DTD $G$ is a TP $Q^{\prime}$ such that (1) for any xML tree $t \in T_{G}, Q^{\prime}(V(t)) \subseteq$ $Q(t)\left(\right.$ resp. $\left.Q^{\prime}(V(t))=Q(t)\right),(2)$ for some $t \in T_{G}, Q^{\prime}(V(t)) \neq \emptyset$. The MCR of $Q$ using $V$ under $G$ is the union of all CRs of $Q$ using $V$ under $G$.

\section{Independence of containing patterns property of tree patterns}

In this section, we show that some classes of tree patterns observe what we call the independence of containing patterns (ICP) property, that is, if a TP is contained in the union of several other TPs, it is contained in one of them.

Theorem 1 Let $P$ and $P_{1}, \ldots, P_{n}$ be tree patterns, in the following cases, if $P \subseteq$ $\bigcup_{i=1}^{n} P_{i}$, then there exists $i \in[1, n]$ such that $P \subseteq P_{i}$.

(1) $P \in P^{\{/, / /,[], *\}}, P_{1}, \ldots, P_{n} \in \widehat{P}^{\{/, / /,[], *\}}$, or

(2) $P \in P^{\{/,[], *\}}, P_{1}, \ldots, P_{n} \in P^{\{/, / /,[], *\}}$.

To prove the above theorem, we need the concept of boolean tree patterns. A boolean pattern $[10]$ is a pattern with no output node. Let $P$ be a boolean pattern. For an XML tree $t$, the result of evaluating $P$ on $t$, denoted $P(t)$, is either TRUE or FALSE. $P(t)$ is TRUE if and only if there is a matching of $P$ in $t$. For two boolean patterns $P_{1}$ and $P_{2}, P_{1} \subseteq P_{2}$ means $P_{1}(t)=$ TRue implies $P_{2}(t)=$ TRue for any xML tree $t$. If $P_{2}$ is in $\widehat{P}^{\{/, / /,[], *\}}$, then $P_{1}(t) \subseteq P_{2}(t)$ iff there is a homomorphism from $P_{2}$ to $P_{1}$ (Recall: a homomorphism is the same as a containment mapping, except it does not need to be output-preserving) [10]. In addition, $P_{1} \cup P_{2}$ returns $P_{1}(t) \vee P_{2}(t)$ for any $t$.

We first prove the following lemma.

Lemma 3 For boolean patterns $P, P_{1}, \ldots, P_{n} \in P^{\{/, / /,[], *\}}$, if $P \in P^{\{/,[], *\}}$, or $P_{1}, \ldots, P_{n} \in$ $\widehat{P}^{\{/, / /,[], *\}}$, then $P \subseteq \bigcup_{i} P_{i}$ implies there exists $i \in[1, n]$ such that $P \subseteq P_{i}$.

Proof We prove the lemma for the case $n=2$. The case $n>2$ is similar.

Using Lemma 1 of [10], we can construct two boolean patterns $Q$ and $Q^{\prime}$ such that $P \subseteq P_{1} \cup P_{2}$ iff $Q \subseteq Q^{\prime} . Q$ and $Q^{\prime}$ are as shown in Fig. 3, where $V$ is a pattern which is contained in both $P_{1}$ and $P_{2}$, and $V$ does not have *-nodes or //-edges (see [10] for how to construct $V$ ). Furthermore, $c$ can be chosen as a label which does not appear 


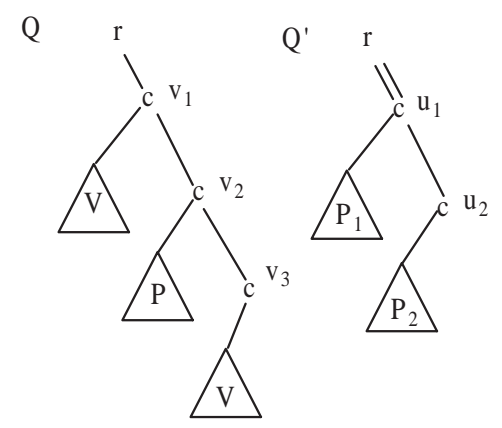

Fig. 3. $Q$ and $Q^{\prime}$ as in [10]

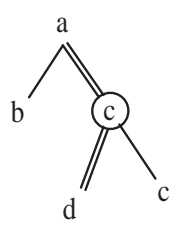

(a)P

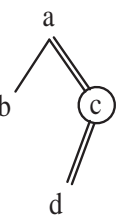

(b) $\mathrm{P} \quad \mathrm{i}$

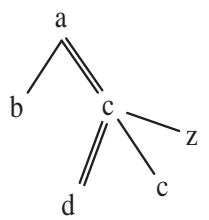

(c)P

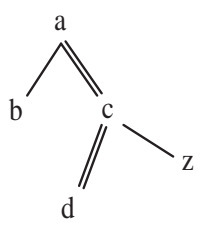

(d) $\mathrm{P} \quad$ i

Fig. 4. Tree patterns $P, P_{i}$ and the boolean patterns $P^{\prime}$ and $P_{i}^{\prime}$

in either $P_{1}$ or $P_{2}$. Note that (1) if $P_{1}, P_{2}$ are in $\widehat{P}^{\{/, / /,[], *\}}$, then $Q^{\prime} \in \widehat{P}^{\{/, / /,[], *\}},(2)$ if $P \in P^{\{/,[], *\}}$, then $Q \in P^{\{/,[], *\}}$. Since $P \subseteq P_{1} \cup P_{2}$ implies $Q \subseteq Q^{\prime}$, by Lemma 1 , we know there is a homomorphism from $Q^{\prime}$ to $Q$. Now examine the structure of $Q$ and $Q^{\prime}$, any homomorphism from $Q^{\prime}$ to $Q$ must map the nodes $u_{1}$ and $u_{2}$ either to $v_{1}$ and $v_{2}$ respectively, or to $v_{2}$ and $v_{3}$ respectively. In the former case, there will be a homomorphism from $P_{2}$ to $P$; in the later case there will be a homomorphism from $P_{1}$ to $P$. Therefore, either $P \subseteq P_{1}$ or $P \subseteq P_{2}$. $\perp$

We are now ready to prove Theorem 1 .

Proof (proof of Theorem 1)

We denote by $P^{\prime}, P_{i}^{\prime}(i \in[1, n])$ the boolean patterns obtained from $P, P_{i}$ by attaching a child node labeled with a distinct label $z$ to the distinguished nodes of $P$ and $P_{i}$ respectively (Since $\Sigma$ is an infinite set of tags, $z$ exists, refer to Fig. 3). Let us denote the new nodes in $P$ and $P_{i}$ by $z_{P}$ and $z_{P_{i}}$ respectively.

We show that $P \subseteq \bigcup_{i \in[1, n]} P_{i}$ implies $P^{\prime} \subseteq \bigcup_{i \in[1, n]} P_{i}^{\prime}$. Let $t$ be any xML tree. For every matching $h$ of $P^{\prime}$ in $t$, there is a matching of $P$ in $t$ which is the one obtained by restricting $h$ to all nodes in $P$ except $z_{P}$. Since $P \subseteq \bigcup_{i \in[1, n]} P_{i}$, there exists an $i \in[1, n]$ such that there is a matching $f$ of some $P_{i}$ in $t$ and $f\left(\operatorname{DN}_{P_{i}}\right)=h\left(\operatorname{DN}_{P}\right)$. This matching $f$ can clearly be extended to $P_{i}^{\prime}$ - simply let $f\left(z_{P_{i}}\right)=h\left(z_{P}\right)$. Therefore $P^{\prime} \subseteq \bigcup_{i \in[1, n]} P_{i}^{\prime}$.

By Lemma 3, there exists $i \in[1, n]$ such that $P^{\prime} \subseteq P_{i}^{\prime}$. Therefore, there is a homomorphism from $P_{i}^{\prime}$ to $P^{\prime}$. This homomorphism implies a containment mapping from $P_{i}$ to $P$. Hence $P \subseteq P_{i} . \perp$ 
Theorem 1 does not hold in the presence of DTDs. For example, consider the DTD in Fig. 1 (a). Under the DTD, $a / / d \subseteq a / b / d \cup a / e / d$. But $a / / d$ is contained in neither $a / b / d$ nor $a / e / d$. Also $a / *$ is contained in the union of $a / g, a / b$ and $a / e$, but it is not contained in any of them.

\section{Equivalent rewritings, single pattern or union of patterns?}

Let $V$ be the view, and $Q$ be the query. By definition, $\operatorname{EMCR}(Q, V) \subseteq Q$. In the best case, $\operatorname{EMCR}(Q, V)$ is equivalent to $Q$ (In this case, we say the MCR of $Q$ using $V$ is equivalent to $Q$ ). The question arises now whether it is possible for $Q$ to have no $\mathrm{ER}$ (which is a single TP) using $V$, but it has a MCR (which is a union of TPs) using $V$ which is equivalent to $Q$. In other words, any single CR of $Q$ using $V$ is not equivalent to $Q$, but the union of all CRs is. We study this problem for the following cases.

\subsection{The class $P^{\{/, / /,[]\}}$}

We first consider the case $V \in P^{\{/, / /,[]\}}, Q \in P^{\{/, / /,[]\}}$and the rewritings of $Q$ using $V$ are all in $P^{\{/, / /,[]\}}$. Using Theorem 1 , we can easily prove the following result.

Theorem 2 Let $V, Q \in P^{\{/, / /,[]\}}$be the view and query respectively. When there are no DTDs, if $Q$ has a MCR using $V$ which is equivalent to $Q$, then it has a single CR using $V$ which is equivalent to $Q$.

Proof Suppose $\operatorname{EMCR}(Q, V)=Q_{1} \circ V \cup \ldots \cup Q_{n} \circ V$, where each $Q_{i}$ is a CR of $Q$ using $V$. Since every $Q_{i} \circ V$ is in $P^{\{/, / /,[]\}}$, if $\operatorname{EMCR}(Q, V)=Q$, then by Theorem $1(1)$ there is $i \in[1, n]$ such that $Q=Q_{i} \circ V$. That is, $Q_{i}$ is an equivalent rewriting of $Q$ using $V$. $\perp$

Because of Theorem 2, for queries and views in $P^{\{/, / /,[]\}}$, if there is no equivalent rewriting of $Q$ using $V$, then no MCR of $Q$ using $V$ is equivalent to $Q$. In other words, if we cannot find a single CR of $Q$ using $V$ which is equivalent to $Q$, then it is impossible to find a union of CRs of $Q$ using $V$ which is equivalent to $Q$. This suggests that we should always use a more efficient algorithm, such as that in [20] to find an equivalent rewriting of $Q$ using $V$.

\subsection{Other classes of tree patterns}

Theorem 2 can be extended to the cases as stated in the next theorem.

Theorem 3 Let $V, Q$ be the view and query respectively. In the following cases, if $Q$ has a MCR using $V$ which is equivalent to $Q$, then it has a single CR using $V$ which is equivalent to $Q$.

(1) $Q \in P^{\{/,[], *\}}$ and there are no leaf ${ }^{*}$-nodes in $Q$.

(2) $V, Q \in \widehat{P}^{\{/, / /,[], *\}}$, and label $\left(\mathrm{DN}_{V}\right) \neq *$.

Note that Theorem $3(2)$ includes the case where $V, Q \in P^{\{/, / /,[]\}}$.

Before proving the above theorem we prove the following lemmas first. 
Lemma 4 Let the query $Q$ and view $V$ be in $P^{\{/, / /,[]\}}$. For every $\mathrm{CR} Q^{\prime} \in P^{\{/, / /,[], *\}}$ of $Q$ using $V$, there is a CR $Q^{\prime \prime} \in P^{\{/, / /,[]\}}$such that $Q^{\prime} \circ V \subseteq Q^{\prime \prime} \circ V$.

Proof By definition of CR, $Q^{\prime} \circ V \subseteq Q$. Since $Q \in P^{\{/, / /,[]\}}$, there is a CM $\delta$ from $Q$ to $Q^{\prime} \circ V . \delta$ partitions $N(Q)$ into two disjoint sets: $N_{1}=\{v \in N(Q) \mid \delta(v) \in N(V)\}$, and $N_{2}=\left\{v \in N(Q) \mid \delta(v) \in N\left(Q^{\prime}\right)-\left\{r t\left(Q^{\prime}\right)\right\}\right\}$. It is clear that for every path $p$ in $Q$, either every node on $p$ is mapped to $V$, or the last node on $p$ which is mapped to $V$, denoted $x$, and the child of $x$ on $p$, denoted $y$, satisfy the following relationship: $\delta(x)$ is on the distinguished path of $V$, and either $(x, y)$ is a //-edge, or $\delta(x)=\mathrm{DN}_{V}$. Furthermore $\mathrm{DN}_{Q}$ is mapped to $\mathrm{DN}_{Q^{\prime} \circ V}=\mathrm{DN}_{Q^{\prime}}$.

If we denote $Q^{\prime \prime}$ the pattern obtained as follows:

(a) Let $r t\left(Q^{\prime \prime}\right)$ be a node labeled with label $\left(\mathrm{DN}_{V}\right)$;

(b) For every path $p$ in $Q$, let the first node on $p$ which is mapped to $Q^{\prime \prime}$ be $y$, and the node preceding $y$ on $p$ be $x$, then add the node subtree $Q_{y}$ under $r t\left(Q^{\prime \prime}\right)$, and connect $r t\left(Q^{\prime \prime}\right)$ and $r t\left(Q_{y}\right)$ with the same type of edge as that of $(x, y)$.

then $Q^{\prime \prime} \in P^{\{/, / /,[]\}}, Q^{\prime \prime} \circ V \subseteq Q$, and $\delta$ makes a CM from $Q^{\prime \prime}$ to $Q^{\prime}$, therefore, $Q^{\prime} \subseteq Q^{\prime \prime}$ and $Q^{\prime} \circ V \subseteq Q^{\prime \prime} \circ V \cdot \perp$

Lemma 5 Let the query $Q$ and view $V$ be in $\widehat{P}^{\{/, / /,[], *\}}$. Suppose label $\left(\mathrm{DN}_{V}\right) \neq *$. For every $\mathrm{CR} Q^{\prime} \in P^{\{/, / /,[], *\}}$ of $Q$ using $V$, there is a $\mathrm{CR} Q^{\prime \prime} \in \widehat{P}^{\{/, / /,[], *\}}$ such that $Q^{\prime} \circ V \subseteq Q^{\prime \prime} \circ V$.

The proof of Lemma 5 is very similar to that of Lemma 4 , except that to ensure $Q^{\prime \prime}$ is in $\widehat{P}^{\{/, / /,[], *\}}$, we need the condition label $\left(\mathrm{DN}_{V}\right) \neq *$. In fact, if this condition is not satisfied, then the lemma does not hold. For example, consider the view $V=a / *[/ c]$, and the query $Q=a / / b$. Clearly $Q^{\prime}=* / / b$ is a CR of $Q$ using $V$. There is no CR $Q^{\prime \prime} \in \widehat{P}^{\{/, / /,[], *\}}$ such that $Q^{\prime} \circ V \subseteq Q^{\prime \prime} \circ V$.

Using the above lemmas, we prove Theorem 3 as follows.

Proof (of Theorem 3) Recall (Section 2.3) that, in both of the cases stated in the theorem, there are CRs $Q_{1}, \ldots, Q_{k}$ of $Q$ using $V$ such that $\operatorname{EMCR}(Q, V)=Q_{1} \circ V \cup \ldots \cup$ $Q_{k} \circ V$. Since $\operatorname{EMCR}(Q, V)=Q$, we know $Q \subseteq Q_{1} \circ V \cup \ldots \cup Q_{k} \circ V$. In case (1), by Theorem 1 (2), there is some $i$ such that $Q \subseteq Q_{i} \circ V$. That is, $Q_{i}$ is an equivalent rewriting of $Q$ using $V$. In case (2), by Lemma 5 , there are $Q_{1}^{\prime}, \ldots, Q_{k}^{\prime} \in \widehat{P}^{\{/, / /,[], *\}}$ such that $Q=Q_{1}^{\prime} \circ V \cup \ldots \cup Q_{k}^{\prime} \circ V$. Clearly each $Q_{i}^{\prime} \circ V$ is a pattern in $\widehat{P}^{\{/, / /,[], *\}}$. By Theorem $1(1)$, there is $i \in[1, k]$ such that $Q \subseteq Q_{i}^{\prime} \circ V$. That is, $Q_{i}^{\prime}$ is an equivalent rewriting of $Q$ using $V . \perp$

We point out that Theorem $3(2)$ does not hold if label $\left(\mathrm{DN}_{V}\right)=*$ and the rewritings are allowed to be in $P^{\{/, / /,[], *\}}$. For example, the query $Q=a / / b$ has the following two CRs using the view $V=a / *: Q_{1}=b$ and $Q_{2}=* / / b$. The union of the expansions of these rewritings is $a / b \cup a / * / / b$, which is equivalent to $Q$. But $Q$ is not equivalent to either $a / b$ or $a / * / / b$.

4.3 Rewritings using multiple individual views

Theorem 2 and Theorem 3 can be easily extended to the case when there are multiple views. 
Theorem 4 Let $V_{1}, \ldots, V_{n}$ be views and $Q$ be a query. In the following cases, if $Q \subseteq$ $\operatorname{EMCR}\left(Q, V_{1}\right) \cup \cdots \cup \operatorname{EMCR}\left(Q, V_{n}\right)$, then there exists $i \in[1, n]$ such that there is an equivalent rewriting of $Q$ using $V_{i}$.

(1) $Q \in P^{\{/,[], *\}}$ and $Q$ has no *-leaves.

(2) $V_{1}, \ldots, V_{n}, Q \in \widehat{P}^{\{/, / /,[], *\}}$, and label $\left(\mathrm{DN}_{V_{i}}\right) \neq *($ for $i \in[1, n])$.

The proof of the above theorem is similar to that of Theorem 3 .

\subsection{Rewritings using intersections of views}

So far we have only considered rewritings using individual views. However, multiple views can be combined to rewrite a query. For instance, when the views have identical root labels and identical labels for distinguished nodes (we say such views are compatible), the intersection of these views may be used to rewrite a query, even though there are no rewritings using any individual view. For example, the query $Q=a[b][c] / d$ does not have a CR using either $V_{1}=a[b] / d$ or $V_{2}=a[c] / d$, but it has a CR using $V_{1} \cap V_{2}$, which is equivalent to $Q$. We will consider this case now.

We need to formally define CR and ER using $V_{1} \cap \cdots \cap V_{n}$ first. We focus on views and queries in the class $P^{\{/, / /,[]\}}$here.

Definition 1 Let $V_{1}, \ldots, V_{n}$ be compatible views in $P^{\{/, / /,[]\}}$, and $Q$ be a query in $P^{\{/, / /,[]\}}$. Suppose $V_{1} \cap \cdots \cap V_{n}$ is not always empty. A contained rewriting (CR) of $Q$ using $V_{1} \cap \cdots \cap V_{n}$ is a TP $Q^{\prime} \in P^{\{/, / /,[]\}}$, such that for any xML tree $t, Q^{\prime}\left(V_{1}(t) \cap\right.$ $\left.\cdots \cap V_{n}(t)\right) \subseteq Q(t)$, and there is at least one $t$ such that $V_{1}(t) \cap \cdots \cap V_{n}(t) \neq \emptyset$. $Q^{\prime}$ is said to be an equivalent rewriting (ER) if $Q^{\prime}\left(V_{1}(t) \cap \cdots \cap V_{n}(t)\right) \supseteq Q(t)$ also holds. The maximal contained rewriting (MCR) of $Q$ using the intersection is the union of all CRs of $Q$ using the intersection.

In the presence of DTD $G$, the CR, MCR and ER are defined similarly, except we consider only XML trees conforming to $G$.

The next example shows that it is possible for a query to have different CRs using the intersection, thus the union of these CRs produces strictly more answers than any single CR.

Example 1 Consider the views $V_{1}=a[x] / b$ and $V_{2}=a[y] / b$, and the query $Q=$ $a[x][y][/ / b / d] / / b[c]$. It can be verified that $Q_{1}=b[c][d], Q_{2}=b[d] / / b[c], Q_{3}=b[/ / b / d][c]$ and $Q_{4}=b[/ / b / d] / / b[c]$ are all CRs of $Q$ using $V_{1} \cap V_{2}$, and none of them is contained in the others.

However, if the union of all CRs becomes equivalent to $Q$, then one of the CRs is equivalent to $Q$. In other words, Theorem 2 can be extended to rewritings using the intersection of compatible views.

Theorem 5 Let $V_{1}, \ldots, V_{n}$ be compatible views in $P^{\{/, / /,[]\}}$, and $Q$ be a query in $P^{\{/, / /,[]\}}$. If the MCR of $Q$ using $V_{1} \cap \cdots \cap V_{n}$ is equivalent to $Q$, then one of the CRs is an $\mathrm{ER}$ of $Q$ using $V_{1} \cap \cdots \cap V_{n}$.

To prove the above result, we need an important property of intersection of TPs, as stated in the following lemma. 
Lemma 6 [14] Let $V_{1}, \ldots, V_{n}$ be compatible views in $P^{\{/, / /,[]\}}$. If $V_{1} \cap \cdots \cap V_{n}$ is not always empty, then there are $\operatorname{TP} s V_{1}^{\prime}, \ldots, V_{k}^{\prime} \in P^{\{/, / /,[]\}}$such that $V_{1} \cap \cdots \cap V_{n}$ is equivalent to $V_{1}^{\prime} \cup \cdots \cup V_{k}^{\prime}$.

We call each $V_{i}^{\prime}$ in the above lemma a disjunctive component of the intersection.

Proof (proof of Theorem 5) By Lemma 6, there are TPs $V_{1}^{\prime}, \ldots, V_{k}^{\prime} \in P^{\{/, / /,[]\}}$such that $V_{1} \cap \cdots \cap V_{n}$ is equivalent to $V_{1}^{\prime} \cup \cdots \cup V_{k}^{\prime}$. Therefore, $Q^{\prime}$ is a CR of $Q$ using $V_{1} \cap \cdots \cap V_{n}$ if and only if it is a CR of $Q$ using every $V_{i}^{\prime}$ for $i=1, \ldots, k$. Suppose $Q_{1}, \ldots, Q_{m}$ are all of the CRs of $Q$ using $V_{1} \cap \cdots \cap V_{n}$. Then the MCR of $Q$ using the intersection is equivalent to $Q$ implies

$$
Q \subseteq \bigcup_{i=1}^{m} \bigcup_{j=1}^{k}\left(Q_{i} \circ V_{j}^{\prime}\right)
$$

Since all TPs involved are in $P^{\{/, / /,[]\}}$, by Theorem 1, we know there is an $i$ and a $j$ such that $Q \subseteq Q_{i} \circ V_{j}^{\prime}$. Therefore, the CR $Q_{i}$ is an equivalent rewriting of $Q$ using $V_{1} \cap \cdots \cap V_{n}$.

Theorem 5 can also be extended to the case where rewritings using both individual views and using intersections of views are considered together. Let $S=\left\{V_{1}, \ldots, V_{n}\right\}$ be a set of compatible views. We use $\operatorname{MCR}(Q, S)(\operatorname{resp} \operatorname{EmcR}(Q, S))$ to denote the union of all CRs (resp. union of the expansions of all CRs) of $Q$ using a single view in $S$ or using the intersection of a subset of views in $S$. Similar to the proof of Theorem 5 , we can prove the theorem below.

Theorem 6 Let $S=\left\{V_{1}, \ldots, V_{n}\right\}$ be compatible views in $P^{\{/, / /,[]\}}$, and $Q$ be a query in $P^{\{/, / /,[]\}}$. If $\operatorname{EMCR}(Q, S)$ is equivalent to $Q$, then there exists a single $\mathrm{CR}, Q^{\prime}$, of $Q$ using either a single view, or using the intersection of some of the views in $S$, such that the expansion of $Q^{\prime}$ is equivalent to $Q$.

\subsection{The presence of DTDs}

Theorem 2 still holds in the presence of a non-recursive schema graph. This is because an equivalent rewriting is also a MCR, and when both $Q$ and $V$ are in $P^{\{/, / /,[]\}}$, the MCR of $Q$ using $V$ under a non-recursive schema graph can be expressed as a single TP in $P^{\{/, / /,[]\}}[6]$.

Theorem 2 does not hold in the presence of recursive schema graphs, as demonstrated by the next example.

Example 2 Consider the query $Q=a / / b$ and the view $V=a / b$ under the recursive DTD in Fig. 1 (b). $Q$ has two CRs: $Q_{1}^{\prime}=b$ and $Q_{2}^{\prime}=b / / b$. The expansions of these CRs are $a / b$ and $a / b / / b$ respectively. Under the DTD, $Q$ is equivalent to the union of $a / b$ and $a / b / / b$, but it is not equivalent to either one of them. That is, there is no single CR of $Q$ using $V$ which is equivalent to $Q$, but the union of the two CRs is equivalent to $Q$. 


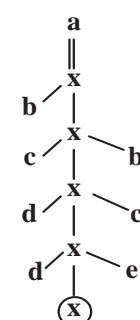

(a) $Q$

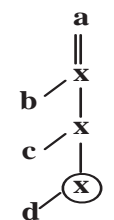

(b) $V$

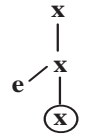

(c) $Q_{1}$

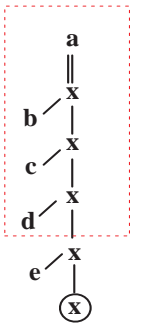

(d) $Q_{1} \circ V$

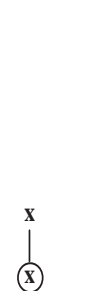

(e) $Q_{2}$

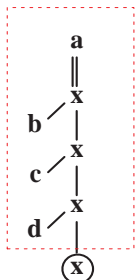

(f) $Q_{2} \circ V$

Fig. 5. $Q$ has no equivalent rewriting using $V$ according to conventional definition, but $Q$ can be fully answered using $V: Q_{1} \circ V \cap Q_{2} \circ V=Q$

Theorem 4 does not hold in the presence of non-recursive schema graphs. For example, consider the DTD $G$ shown in Fig. 1 (a). Under the DTD, if we let $Q=a / / d$, $V_{1}=a / b / d$ and $V_{2}=a / e / d$, then $\operatorname{MCR}\left(Q, V_{1}\right)=V_{1}$ and $\operatorname{MCR}\left(Q, V_{2}\right)=V_{2}$, and $\operatorname{MCR}\left(Q,\left\{V_{1}, V_{2}\right\}\right)=V_{1} \cup V_{2}={ }_{G} Q$. But $Q$ is not equivalent to either $V_{1}$ or $V_{2}$ under $G$.

Theorem 5 still holds in the presence of non-recursive DTDs. This is because, under a non-recursive DTD $G, V_{1} \cap \cdots \cap V_{n}$ is equivalent to a single TP in $P^{\{/, / /,[]\}}$, say $V$. As given in [6], the MCR of $Q$ using $V$ under $G$ is contained in a single CR of $Q$ using $V$ under $G$. Therefore, the MCR of $Q$ using $V_{1} \cap \cdots \cap V_{n}$ under $G$ is contained in a single CR, say $Q^{\prime}$, of $Q$ using $V_{1} \cap \cdots \cap V_{n}$ under $G$. If the MCR produces all answers to $Q$, namely $Q$ is contained in the MCR, then $Q^{\prime}$ is an ER of $Q$.

\subsection{Discussion: answerability of $Q$ using $V$}

In this section, we show, by example, that even if $Q$ has no equivalent rewriting using $V$ according to the definition given in Section 2, it is still possible to answer $Q$ completely using $V$. The next example demonstrates this point.

Example 3 Consider the query $Q$ and view $V$ shown in Fig. 5 (a) and (b) respectively. $Q$ has no equivalent rewriting using $V$. But given any xML tree $t$, we can find $Q(t)$ using the view as follows. We evaluate the query $Q_{1}=x / x[e] / y$ over the subtrees in $V(t)$, and denote the results as $Q_{1}(V(t))$; we then evaluate $Q_{2}=x / y$ over the subtrees in $V(t)$, and obtaining a set denoted as $Q_{2}(V(t))$. Finally, we take the intersection of $Q_{1}(V(t))$ and $Q_{2}(V(t))$. It can be verified that $Q(t)=Q_{1}(V(t)) \cap Q_{2}(V(t))$.

\section{Redundant views}

In this section we assume there are multiple views $V_{1}, \ldots, V_{n}$ in $\widehat{P}^{\{/, / /,[], *\}}$. We now ask the question when a subset of views $V_{i_{1}}, \ldots, V_{i_{k}}$ are redundant in the sense that for every query $Q$, the MCRs of $Q$ using $V_{i_{1}}, \ldots, V_{i_{k}}$ are all contained in the union of the MCRs of $Q$ using the other views. Formally we have 
Definition 2 Let $V_{1}, \ldots, V_{n} \in \widehat{P}^{\{/, / /,[], *\}}$ be views and $k<n$. If for every TP $Q \in$ $\widehat{P}^{\{/, / /,[], *\}}$,

$$
\bigcup_{i=1}^{k} \operatorname{EMCR}\left(Q, V_{i}\right) \subseteq \bigcup_{j=k+1}^{n} \operatorname{EMCR}\left(Q, V_{j}\right)
$$

then we say the views $V_{1}, \ldots, V_{k}$ are redundant.

Intuitively, when considering CRs the redundant views can be ignored because all answers returned by CRs using the redundant views can be returned by CRs using other views.

One might wonder how the redundant views problem is related to the view containment problem $\cup_{i=1}^{k} V_{i} \subseteq \cup_{j=k+1}^{n} V_{j}$. As we show in the next example, the condition $\cup_{i=1}^{k} V_{i} \subseteq \cup_{j=k+1}^{n} V_{j}$ is neither sufficient nor necessary for $V_{1}, \ldots, V_{k}$ to be redundant.

Example 4 (1) Let $V_{1}=a[b] / c$ and $V_{2}=a / c$. Clearly $V_{1} \subseteq V_{2}$. But $V_{1}$ is not redundant, because the query $Q=a[b] / c / d$ has a CR using $V_{1}$, but it does not have a CR using $V_{2}$. (2) Now let $V_{1}=a / x / x$ and $V_{2}=a / x$. It is easy to verify $V_{1} \nsubseteq V_{2}$, but $V_{1}$ is redundant.

We now provide the following sufficient and necessary condition for redundant views.

Theorem 7 Given $V_{1}, \ldots, V_{n} \in P^{\{/, / /,[], *\}}, V_{1}, \ldots, V_{k}(k<n)$ are redundant iff for every $i \in[1, k]$, the union of the expansions of the CRs of $V_{i}$ using the views $V_{k+1}, \ldots, V_{n}$ is equivalent to $V_{i}$, that is,

$$
V_{i}=\bigcup_{j=k+1}^{n} \operatorname{EMCR}\left(V_{i}, V_{j}\right)
$$

Proof (only if) we only need to consider the query $Q=V_{i}$ for $i \in[1, k]$. Clearly there is an equivalent rewriting of $V_{i}$ using itself. Therefore $\bigcup_{j=1}^{k} \operatorname{EMCR}\left(V_{i}, V_{j}\right)=V_{i}$ for $i \in[1, k]$. By definition, $V_{1}, \ldots, V_{k}$ are redundant implies that

$$
\bigcup_{j=1}^{k} \operatorname{EMCR}\left(V_{i}, V_{j}\right) \subseteq \bigcup_{j=k+1}^{n} \operatorname{EMCR}\left(V_{i}, V_{j}\right) .
$$

Thus

$$
V_{i} \subseteq \bigcup_{j=k+1}^{n} \operatorname{EMCR}\left(V_{i}, V_{j}\right)
$$

for all $i \in[1, k]$. Since $\operatorname{EMCR}\left(V_{i}, V_{j}\right) \subseteq V_{i}$ for all $j \in[k+1, n]$, we know

$$
V_{i}=\bigcup_{j=k+1}^{n} \operatorname{EMCR}\left(V_{i}, V_{j}\right)
$$

(if) Suppose for every $i \in[1, k]$,

$$
V_{i}=\bigcup_{j=k+1}^{n} \operatorname{EMCR}\left(V_{i}, V_{j}\right)
$$


Suppose $\operatorname{EMCR}\left(V_{i}, V_{j}\right)=Q_{j, 1} \circ V_{j} \cup \ldots \cup Q_{j, m_{j}} \circ V_{j}$. Then

$$
V_{i}=\bigcup_{j=k+1}^{n} \bigcup_{s=1}^{m_{j}}\left(Q_{j, s} \circ V_{j}\right)
$$

For any TP $Q$, if $Q^{\prime}$ is a CR of $Q$ using $V_{i}$, then $Q^{\prime} \circ V_{i} \subseteq Q$. Hence

$$
Q^{\prime} \circ \bigcup_{j=k+1}^{n} \bigcup_{s=1}^{m_{j}}\left(Q_{j, s} \circ V_{j}\right) \subseteq Q
$$

i.e.,

$$
\bigcup_{j=k+1}^{n} \bigcup_{s=1}^{m_{j}}\left(\left(Q^{\prime} \circ Q_{j, s}\right) \circ V_{j}\right) \subseteq Q
$$

Thus every $Q^{\prime} \circ Q_{j, s}\left(s=1, \ldots, n_{j}\right)$, if not empty, is a CR of $Q$ using $V_{j}$. Therefore,

$$
Q^{\prime} \circ V_{i} \subseteq \bigcup_{j=k+1}^{n} \operatorname{EMCR}\left(Q, V_{j}\right)
$$

Since $Q^{\prime}$ is an arbitrary CR of $Q$ using $V_{i}$, we know

$$
\bigcup_{i=1}^{k} \operatorname{EMCR}\left(Q, V_{i}\right) \subseteq \bigcup_{j=k+1}^{n} \operatorname{EMCR}\left(Q, V_{j}\right) \text {. }
$$

By definition, $V_{1}, \ldots, V_{k}$ are redundant. $\perp$

If the views $V_{1}, \ldots, V_{k}$ are in $P^{\{/,[], *\}}$ and they have no *-leaves, or if $V_{1}, \ldots, V_{n}$ are in $\widehat{P}^{\{/, / /,[], *\}}$ and the distinguished nodes of $V_{k+1}, \ldots, V_{n}$ are not labeled $*$, then by Theorem $4, V_{i}=\bigcup_{j=k+1}^{n} \operatorname{EMCR}\left(V_{i}, V_{j}\right)$ iff there is $j \in[k+1, n]$ such that $V_{i}$ has an equivalent rewriting using $V_{j}$. This leads to the following corollary.

Corollary 1 Given views $V_{1}, \ldots, V_{n}$, In the following cases, $V_{1}, \ldots, V_{k}(k<n)$ are redundant iff for every $i \in[1, k]$, there exists $j \in[k+1, n]$ such that $V_{i}$ has an equivalent rewriting using $V_{j}$.

(1) $V_{1}, \ldots, V_{k}$ are in $P^{\{/,[], *\}}$ and they have no *-leaves.

(2) $V_{1}, \ldots, V_{n}$ are in $\widehat{P}^{\{/, / /,[], *\}}$, and the distinguished nodes of $V_{k+1}, \ldots, V_{n}$ are not labeled *.

Note that case (2) above includes the case where $V_{1}, \ldots, V_{n}$ are in $P^{\{/, / /,[]\}}$.

Theorem 7 still holds when there is a schema graph, and the proof is similar. However, when a schema graph exists, the condition in Corollary 1 is still sufficient but no longer necessary. The proof of sufficiency is similar to the case when there are no schema graphs. The non-necessity is shown by the DTD in Fig. 1 (a), and the views $V_{1}=a / b, V_{2}=a / e$, and $V_{3}=a / / d$. Clearly $V_{3}$ is redundant under the DTD, but it does not have an equivalent rewriting using either $V_{1}$ or $V_{2}$.

A special case of Corollary 1 is when all the views $V_{1}, \ldots, V_{n}$ are in $P^{\{/, / /,[]\}}$, and they are compatible, that is, their distinguished nodes have identical labels. In this case, if $V_{i}$ is redundant, then by Corollary 1 , there is $j \neq i$ such that $V_{i}$ has an equivalent rewriting using $V_{j}$. That is, there is a TP $Q^{\prime}$ such that $V_{i}=Q^{\prime} \circ V_{j}$. If the distinguished path of $V_{i}$ does not have repeating labels, then we know the root of $Q^{\prime}$ must be the same as the distinguished node of $Q^{\prime}$. Therefore $V_{i} \subseteq V_{j}$. This proves the following corollary. 
Corollary 2 Let $V_{1}, \ldots, V_{n}$ be compatible views in $P^{\{/, / /,[]\}}$. If $V_{1}, \ldots, V_{k}(k<n)$ are redundant, then for every $i \in[1, k]$ such that the distinguished path of $V_{i}$ has no repeating labels, there exists $j \in[k+1, n]$ such that $V_{i} \subseteq V_{j}$.

The condition that the distinguished path of $V_{i}$ has no repeating labels is important in the above corollary. If it is not satisfied, the corollary does not hold. This is easily seen in Example 4 (2).

Identifying redundant views Corollary 1 provides a means to find the redundant views. To see whether $V_{i}$ is redundant we only need to check whether there exists $V_{j}$ such that $V_{i}$ has an equivalent rewriting using $V_{j}$. To do so we can use the algorithm in $[20]$.

5.1 Redundant views when intersections of views are used for rewriting

We now re-examine the redundant views problem, taking into consideration rewritings using intersections of views as well as individual views.

Let $S=\left\{V_{1}, \ldots, V_{n}\right\}$ be a set of compatible views. We use $\operatorname{EMCR}(Q, S)$ to denote the union of expansions of all CRs of $Q$ using any single view in $S$ or using the intersection of any subset of views in $S$. The new meaning of redundant views is as follows.

Definition 3 Let $S=\left\{V_{1}, \ldots, V_{n}\right\}$ be a set of compatible views and $S^{\prime}$ be a proper subset of $S$. We say that $S^{\prime}$ is strongly redundant if for every query $Q, \operatorname{EMCR}(Q, S) \subseteq$ $\operatorname{EMCR}\left(Q, S-S^{\prime}\right)$.

The following theorem provides a necessary condition for $S^{\prime}$ to be strongly redundant.

Theorem 8 Let $S=\left\{V_{1}, \ldots, V_{n}\right\}$ be a set of compatible views in $P^{\{/, / /,[]\}}$and $S^{\prime}$ be a proper subset of $S$. If $S^{\prime}$ is strongly redundant, then for every view $V \in S^{\prime}$, $V=\operatorname{EMCR}\left(V, S-S^{\prime}\right)$

Proof We prove the theorem by contradiction. Suppose there is $V \in S^{\prime}$ such that $V \neq$ $\operatorname{EMCR}\left(V, \mathcal{S}-\mathcal{S}^{\prime}\right)$. That is, $V \nsubseteq \operatorname{EMCR}\left(V, \mathcal{S}-\mathcal{S}^{\prime}\right)$. Consider the query $Q=V$. $Q$ has an equivalent rewriting using $S$, whose expansion is $V$ itself. Thus $\operatorname{EMCR}(Q, S)=V$. Therefore $\operatorname{EMCR}(Q, S) \nsubseteq \operatorname{EMCR}\left(Q, \mathcal{S}-\mathcal{S}^{\prime}\right)$. This contradicts the assumption that $S^{\prime}$ is strongly redundant. $\perp$

However, the condition that $\forall V \in S^{\prime}, V=\operatorname{EMCR}\left(V, S-S^{\prime}\right)$ is generally not sufficient for $S^{\prime}$ to be strongly redundant, as shown in the following example.

Example 5 Let $V$ be the view shown in Fig. 5 (b), $V_{1}$ be the pattern shown in Fig. 5 (d), and $V_{2}$ be the pattern shown in Fig. 5 (f). Let $S=\left\{V, V_{1}, V_{2}\right\}$ and $S^{\prime}=\left\{V_{1}, V_{2}\right\}$. As shown in Fig. 5, $V_{1}=Q_{1} \circ V$ and $V_{2}=Q_{2} \circ V$. However, $S^{\prime}$ is not strongly redundant because there is $Q$ (as shown in Fig. 5 (a)) which has an equivalent rewriting using $V_{1} \cap V_{2}$, but no equivalent rewriting using $V$.

The next theorem provides a sufficient condition for $S^{\prime}$ to be strongly redundant. 
Theorem 9 Let $S=\left\{V_{1}, \ldots, V_{n}\right\}$ be a set of compatible views in $P^{\{/, / /,[]\}}$and $S^{\prime}$ be a proper subset of $S$. If for every view $V \in S^{\prime}, V=\operatorname{EMCR}\left(V, S-S^{\prime}\right)$, and for every intersection I involving views in $S^{\prime}$ each disjunctive component $V^{\prime}$ in I satisfies $V^{\prime}=\operatorname{EMCR}\left(V^{\prime}, S-S^{\prime}\right)$, then $S^{\prime}$ is strongly redundant.

Proof Let $Q$ be any TP in $P^{\{/ / / /,[]\}}$. Suppose $Q$ has a CR $Q^{\prime}$ using the intersection $I$ of some views (a single view is treated as a special intersection). Suppose $I=$ $V_{1}^{\prime} \cup \ldots \cup V_{k}^{\prime}$, then $Q^{\prime}$ is a CR of $Q$ using $V_{i}^{\prime}$ for all $i \in[1, k]$. By assumption, every $V_{i}^{\prime}$ satisfies $V_{i}^{\prime}=\operatorname{EMCR}\left(V_{i}^{\prime}, S-S^{\prime}\right)$. By Theorem 6 , there is intersection $I_{i}$ of some views in $S-S^{\prime}$ such that $V_{i}^{\prime}$ has an equivalent rewriting using $I_{i}$. Let $V_{i}^{\prime}=Q_{i} \circ I_{i}$. Then $Q^{\prime} \circ\left(Q_{i} \circ I_{i}\right) \subseteq Q$ (hence $\left.\left(Q^{\prime} \circ Q_{i}\right) \circ I_{i} \subseteq Q\right)$. Thus $Q^{\prime} \circ Q_{i}$ is a CR of $Q$ using $I_{i}$, and $Q^{\prime} \circ I=Q^{\prime} \circ\left(V_{1}^{\prime} \cup \ldots \cup V_{k}^{\prime}\right)=\bigcup_{i=1}^{k}\left(Q^{\prime} \circ Q_{i}\right) \circ I_{i}$. Therefore, $\operatorname{EMCR}(Q, S) \subseteq$ $\operatorname{EMCR}\left(Q, S-S^{\prime}\right)$. By definition, $S^{\prime}$ is strongly redundant. $\perp$

\section{Related work}

When a tree pattern is viewed as a constraint over XML trees, the independence of containing patterns property can be regarded as a special case of the independence of negative constraints (INC) property: given constraints $C, C_{1}, \ldots, C_{n}, C$ implies $C_{1} \vee$ $\cdots \vee C_{n}$ iff $C$ implies some $C_{i}$. The INC property was first studied in [7] and since then found to hold for many classes of constraints. For works on tree pattern query rewriting using views, besides the papers [6,20,9] discussed in Section 1, several other papers have dealt with with the problem. In particular, [19] extended the work of [6] to tree patterns in $\widehat{P}^{\{/, / /,[], *\}}$, and presented efficient algorithms to find maximal contained rewritings when there is no schema graph or when there is an acyclic schema graph. [18] considered rewritings using different combinations of multiple views, one of them is intersection. [14] studied the problem of query answerability using views for general XPATH queries (that may involve negation, and disjunction), that is, given $Q$ and $V_{1}, \cdots, V_{n}$, whether there are $Q_{1}, \cdots, Q_{n}$ such that $Q_{1} \circ V_{1} \cup \cdots \cup Q_{n} \circ V_{n}=Q$. As shown in Section 4 (Theorem 4 ), when $Q$ and $V_{1}, \cdots, V_{n}$ are restricted to some classes of tree patterns, the problem is significantly simplified because no unions of tree patterns need to be considered, hence the simple algorithm in [20] can be applied. [15]defined correct rewritings of TPs, using a single view, for tree patterns with multiple output nodes. The rewriting is essentially a mapping from the output nodes of $Q$ to the output nodes of $V$ under which $V \subseteq Q$. When restricted to a single output node for each pattern or view, the mapping is unique, and the existence of a correct rewriting simply means $V \subseteq Q$. [3] addressed the problem of answering XPATH queries using a single materialized view where, for the view, a combination of node references, typed data values, and full paths may be stored. However, the way in which a query is answered using the view is different: one can follow node references to go to the original document, so the original XML tree cannot be discarded. [16] presented an algorithm for equivalently answering XPATH queries using multiple materialized views based on the assumption that the Dewey codes are stored in the materialized views so that the common ancestors of nodes in different views can be found. [2] studied a different type of equivalent rewriting using multiple views in the presence of structural summaries and integrity constraints: the answer sets of the views are nodes rather than subtrees, and the answers to the new query are obtained by combining answers to the views through a number of algebraic operations. There have also been works on rewriting XQuery 
queries using views $[13,4,21]$. In relational query rewriting using views, the redundant views problem was studied in [8], which showed similar properties for views which are redundant: a view is redundant if and only if it has an equivalent rewriting using the other views. We are not aware of any work on the form of equivalent rewritings, neither for XPATH rewritings nor for relational rewritings.

\section{Conclusion}

We showed that some tree patterns observe the independence of containing patterns property. Based on which, we showed that for some classes of tree patterns, the equivalent rewriting using views can be expressed as a single tree pattern rather than the union of multiple tree patterns. We also identified necessary and sufficient conditions for a subset of views to be redundant. In doing this, we considered different scenarios: the absence or presence of DTDs, rewritings using multiple single views, and rewritings using the intersection of views.

Acknowledgement This work is partially supported by grant from the Research Grant Council of the Hong Kong Special Administrative Region, China (CUHK418205).

\section{References}

1. S. Amer-Yahia, S. Cho, L. V. S. Lakshmanan, and D. Srivastava. Minimization of tree pattern queries. In SIGMOD, pages 497-508. ACM, 2001.

2. A. Arion, V. Benzaken, I. Manolescu, and Y. Papakonstantinou. Structured materialized views for XML queries. In $V L D B$, pages 87-98. ACM, 2007.

3. A. Balmin, F. Özcan, K. S. Beyer, R. Cochrane, and H. Pirahesh. A framework for using materialized XPath views in XML query processing. In $V L D B$, pages 60-71. Morgan Kaufmann, 2004.

4. A. Deutsch and V. Tannen. Reformulation of XML queries and constraints. In ICDT, pages 225-241. Springer, 2003.

5. A. Y. Halevy. Answering queries using views: A survey. VLDB J., 10(4):270-294, 2001.

6. L. V. S. Lakshmanan, H. Wang, and Z. J. Zhao. Answering tree pattern queries using views. In $V L D B$, pages $571-582$. ACM, 2006.

7. J.-L. Lassez and K. McAloon. Independence of negative constraints. In TAPSOFT, Vol.1, pages 19-27. Springer, 1989.

8. C. Li, M. Bawa, and J. D. Ullman. Minimizing view sets without losing query-answering power. In ICDT, pages 99-113. Springer, 2001.

9. B. Mandhani and D. Suciu. Query caching and view selection for XML databases. In $V L D B$, pages $469-480$. ACM, 2005.

10. G. Miklau and D. Suciu. Containment and equivalence for an XPath fragment. In PODS, pages 65-76. ACM, 2002.

11. G. Miklau and D. Suciu. Containment and equivalence for a fragment of XPath. J. ACM, $51(1), 2004$.

12. A. Nash, L. Segoufin, and V. Vianu. Determinacy and rewriting of conjunctive queries using views: A progress report. In ICDT, pages 59-73. Springer, 2007.

13. N. Onose, A. Deutsch, Y. Papakonstantinou, and E. Curtmola. Rewriting nested XML queries using nested views. In SIGMOD, pages 443-454. ACM, 2006.

14. K. Tajima and Y. Fukui. Answering XPath queries over networks by sending minimal views. In $V L D B$, pages 48-59. Morgan Kaufmann, 2004.

15. J. Tang and S. Zhou. A theoretic framework for answering XPath queries using views. In XSym, pages 18-33. Springer, 2005.

16. N. Tang, J. X. Yu, M. T. Özsu, B. Choi, and K.-F. Wong. Multiple materialized view selection for XPath query rewriting. In ICDE, pages 873-882. IEEE, 2008.

17. J. Wang, R. W. Topor, and M. J. Maher. Rewriting union queries using views. Constraints, 10(3):219-251, 2005. 
18. J. Wang and J. X. Yu. Xpath rewriting using multiple views. In DEXA, pages 493-507. Springer, 2008.

19. J. Wang, J. X. Yu, and C. Liu. Contained rewritings of xpath queries using views revisited. In WISE, pages 410-425. Springer, 2008.

20. W. Xu and Z. M. Özsoyoglu. Rewriting XPath queries using materialized views. In VLDB, pages 121-132. ACM, 2005.

21. C. Yu and L. Popa. Constraint-based XML query rewriting for data integration. In SIGMOD, pages 371-382. ACM, 2004. 